\title{
Complejo de esclerosis tuberosa: perfil neuropsicológico y propuesta de intervención
}

\section{Tuberous Sclerosis Complex: neuropsychological profile and intervention proposal}

\author{
Luisa Fernanda Bernal Botero ${ }^{1}$ (D), Yaira Zuleine Arias-Ramírez ${ }^{1}$ (D), \\ Cesar Mauricio Pineda Graciano ${ }^{2}$ (D). \\ ${ }^{1}$ Facultad de Psicología; Universidad de San Buenaventura; Medellin; Colombia. \\ ${ }^{2}$ Gestión del conocimiento; Instituto de capacitación Los Álamos; Itagüi; Colombia.
}

Correspondencia

$\checkmark$ lfbernalbotero@gmail.com

\section{Cómo citar}

Bernal Botero LF, Arias-Ramírez YZ, Pineda Graciano CM. Complejo de esclerosis tuberosa: perfil neuropsicológico y propuesta de intervención. Rev. Investig. Innov. Cienc. Salud. 2020;2(1): 98-115. https://doi.org/ 10.46634/riics.46

Recibido: $10 / 04 / 2020$

Revisado: $28 / 04 / 2020$

Aceptado: $15 / 05 / 2020$

Editor

Jorge Mauricio Cuartas Arias, Ph.D. (iD

\section{Coeditor}

Fraidy-Alonso Alzate-Pamplona, MSc. ic

\section{Corrección de estilo}

Nicolasa Marín González

Copyright (C) 2020. Fundación Universitaria María Cano. La Revista de Investigación e Innovación en Ciencias de la Salud proporciona acceso abierto a todo su contenido bajo los términos de la licencia creative commons Atribución-NoComercial-SinDerivadas 4.0 Internacional (CC BY-NC-ND 4.0).

\section{Conflicto de intereses}

Los autores han declarado que no hay conflicto de intereses.

\section{Disponibilidad de datos}

Todos los datos relevantes se encuentran en el artículo. Para información más detallada, escribir al autor de correspondencia.

\section{Fondos}

No. Esta investigación no recibió ninguna subvención específica de agencias de financiamiento en los sectores público, comercial o sin fines de lucro.

\section{Resumen}

El Complejo de Esclerosis Tuberosa (CET) es un trastorno genético de herencia autosómica dominante causado por la mutación en uno de los genes TSC1 o TSC2. Los pacientes con una afectación CET grave de tipo neurológica posiblemente presentarán epilepsia, discapacidad intelectual, problemas específicos del aprendizaje y trastornos de la conducta, por lo que la evaluación neuropsicológica en individuos con esta patología cobra un carácter importante al proporcionar información sobre los déficits cognitivos que subyacen en la afectación cerebral, que alteran el funcionamiento intelectual y los aspectos adaptativos. El actual trabajo presenta el perfil de una paciente adulta femenina con antecedente de CET, epilepsia y discapacidad intelectual, así como la descripción de una propuesta de intervención neuropsicológica basada en el funcionamiento ejecutivo dorsolateral.

\section{Palabras clave}

Complejo de Esclerosis Tuberosa; epilepsia; déficit cognitivo; discapacidad intelectual; evaluación neuropsicológica.

\section{Abstract}

Tuberous Sclerosis Complex (TSC) is an autosomal dominant inherited genetic disorder caused by mutation in one of the TSC1 or TSC2 genes. Patients with severe neurological-type CET involvement may have epilepsy, intellectual disability, specific learning problems, and behavioral disorders. For this reason, the neuropsychological evaluation in individuals with this pathology becomes an important character by providing information on the cognitive deficits that underlie brain involvement that alter intellectual functioning and adaptive aspects. The current work presents the cognitive profile of a female adult patient with a history of TSC, epilepsy and intellectual disability and the description of a proposed neuropsychological intervention based on dorsolateral executive functioning.

\section{Keywords}

Tuberous Sclerosis Complex; epilepsy; cognitive deficit; intellectual disability; neuropsychological evaluation. 


\section{Introducción}

El Complejo de Esclerosis Tuberosa (CET) es un trastorno genético de herencia autosómica dominante causado por la mutación en uno de los genes TSG1 o TSC2. Principalmente, se caracteriza por irregularidades en el desarrollo cerebral y la aparición de tumores benignos en varios órganos del cuerpo como el cerebro, médula espinal, retina, piel, pulmones, riñones y corazón, que se pueden presentar en cualquier momento de la vida a través de la presencia de crisis epilépticas asociadas a alteraciones cutáneas [1]. Los pacientes con una afectación CET grave de tipo neurológica pueden presentar, además de epilepsia, Discapacidad Intelectual (DI), problemas específicos del aprendizaje y trastornos de la conducta.

El Coeficiente Intelectual (CI) medido a través de una prueba psicométrica en pacientes con CET es altamente variable; sin embargo, se establece que la presencia de Discapacidad Intelectual (DI) es mayor en mutaciones del gen TSC2 y en pacientes con antecedente de epilepsia, principalmente si tuvieron espasmos infantiles antes del primer año de vida [2].

Este estudio tuvo como objetivo determinar cuáles son las características del funcionamiento cognitivo y las posibilidades de intervención neuropsicológica en una paciente con diagnóstico de CET. Entre las especificidades, se planteó describir el perfil cognitivo de la paciente e identificar los puntos de encuentro o las diferencias entre la valoración de su perfil neuropsicológico y lo reportado en la evidencia científica. A su vez, se propuso un plan de rehabilitación neuropsicológica acorde con la valoración inicial, su funcionamiento cotidiano y las necesidades en su contexto. La investigación fue llevada a cabo bajo la metodología de estudio de caso, que resulta particularmente apropiada dado el notable grado de intensidad en el análisis de los datos heterogéneos (información proveniente de entrevistas, observación directa, evaluación y análisis de pruebas neuropsicológicas e indagación teórica sobre el fenómeno estudiado) y el reducido período de presentación frente al paciente. Para dar respuesta a las conjeturas de la investigación, se desarrolló una evaluación neuropsicológica desde el enfoque empírico analítico que permitió valorar el perfil cognitivo de un adulto con CET, epilepsia y DI. La paciente se encuentra en modalidad de hogar sustituto, bajo la medida de restauración de derechos por parte del Instituto Colombiano de Bienestar Familiar (ICBF), en el Instituto de Capacitación los Álamos, en Medellín, Colombia. Cabe resaltar que, de acuerdo con la literatura revisada y particularmente en Colombia, hay pocas investigaciones sobre este síndrome desde disciplinas como la neurología y neuropsicología a nivel mundial. A pesar de que se han encontrado algunos estudios de caso sobre las manifestaciones clínicas del CET, no hay datos significativos sobre los índices de incidencia y prevalencia de este síndrome, por lo tanto, no hay suficientes estudios neuropsicológicos que describan las dificultades cognoscitivas presentes en el complejo. Por esta razón, el abordaje de esta investigación desde una metodología empírico-analítica (sobre un caso específico) es pertinente, ya que contribuye con evidencias que amplían su cuerpo teórico y metodológico desde el campo de las neurociencias, especialmente la neuropsicología, pues esta se encarga de estudiar los efectos de un daño, lesión o malformación en el Sistema Nervioso Central (SNG) y su impacto en el funcionamiento de los procesos cognitivos, emocionales y comportamentales de un individuo.

De acuerdo con lo descrito anteriormente, surge la necesidad de profundizar, mediante el estudio de caso, el perfil cognoscitivo de la paciente. Se propone hacerlo a través de la evaluación neuropsicológica y con el fin de revisar y analizar las mediciones, describiendo los puntajes mínimos, máximos y promedios, en todas las variables del funcionamiento cognitivo, de cada proceso. La paciente con la cual se desarrolló este estudio de caso se identificará como B. 


\section{Fundamentación teórica}

El CET es un trastorno multisistémico que exhibe un patrón de herencia autosómico dominante, caracterizado por la presencia de crecimiento de hamartomas en diferentes órganos debido a una mutación heterocigota en uno de los dos genes supresores de tumores. La sintomatología de la enfermedad se expresa de manera heterogénea según el grado de lesión y usualmente hace su aparición desde la infancia. Según Boronat, Sábado, y Vendrell [1,p6] "se representa principalmente por un trastorno del desarrollo cerebral y la aparición de tumores benignos en muchos órganos del cuerpo". Las complicaciones pueden incluir DI, crisis epilépticas incontrolables, tumores cerebrales, del corazón y renales. Más del $90 \%$ de pacientes afectados presentará las lesiones típicas del $\mathrm{SNC}$, que consisten en nódulos subependimario y túberes con crecimiento progresivo. Los pacientes con una afectación CET grave de tipo neurológica posiblemente presentarán epilepsia, DI, problemas específicos del aprendizaje y trastornos de la conducta.

La epilepsia secundaria al CET es la causa más frecuente y significativa de morbilidad en este cuadro clínico: afecta casi del 79\% al 90\% de los pacientes; su aparición se observa en los primeros años de vida del individuo; su presentación más frecuente son los espasmos infantiles seguidos de crisis parciales simples, parciales complejas o parciales con generalización secundaria [3]. El déficit cognitivo es una de las presentaciones primarias del CET: afecta del $44 \%$ al $65 \%$ de los pacientes. Se ha establecido la asociación entre los espasmos infantiles, epilepsia fármaco resistente y los hamartomas glioneurales corticales [3]. Según Boronat et al. [1,p9] "La prevalencia de Discapacidad Intelectual en CET se estima entre 50 - 80\% entre los afectados; así como otros trastornos de índole psiquiatrica (trastorno de ansiedad, depresión y estrés post-traumático)".

En Colombia no se conoce la frecuencia real de esta entidad, pues no se tienen cifras que permitan conocer el porcentaje de pacientes con esta afectación (CET) que además tienen DI. Asimismo, por ser una patología poco frecuente, los estudios a nivel mundial indican que tiene una prevalencia de 1 de cada 5000 a 10000 nacidos vivos y, de estos, dos tercios de los casos se presentan de manera esporádica. En países como Estados Unidos se cree que hay una prevalencia de 1 caso por cada 6000 a 10000 personas. En Latinoamérica existen pocos datos de prevalencia, sin embargo, se estima que en países como Brasil tiene una incidencia de 1 por 10 mil a 50 mil nacidos [4].

Por otra parte, cabe mencionar que cualquier condición que comprometa el SNG durante su desarrollo puede producir DI. Según el Manual Diagnóstico y estadístico de los trastornos mentales (DSM5), se le denomina DI al trastorno del desarrollo intelectual que se caracteriza "por limitaciones importantes en el funcionamiento del intelecto y en el comportamiento adaptativo que incluye habilidades prácticas y sociales de la vida diaria, y debe originarse antes de los 18 años" [5,p329].

La Asociación Americana de Discapacidad Intelectual y de Desarrollo (AAIDD), a través de un consenso entre expertos, agrupó una serie de posibles causas, entre las más comunes: factores genéticos, ya sea por genes recesivos autonómicos, anormalidades multigenéticas de expresión variable o aberraciones cromosómaticas; desórdenes adquiridos durante los procesos prenatal, perinatal o posnatal y problemas relacionados con afecciones psiquiátricas como trastornos generalizados del desarrollo [6].

El DSM5 plantea una prevalencia global en DI de aproximadamente el 1\% de la población en general y las tasas varían según la edad. Los datos epidemiológicos sobre la DI presentan grandes diferencias en la prevalencia de estimación [7]. Los estudios que únicamente se basan en la estimación del CI para establecer el diagnóstico de DI, hallan tasas de prevalencia 
más cercanas al 3\%, mientras que los estudios que, además del CI, incluyen limitaciones en la conducta adaptativa, encuentran tasas más cercanas al 1\% [8]. Igualmente, los estudios que hacen uso de información basada en registros nacionales o de centros de atención a la discapacidad estiman tasas de prevalencia inferiores a las ya descritas, lo cual podría indicar que hay un número considerable de personas que cumplen con criterios de DI, pero no han sido identificados oficialmente [9].

Por lo anterior, la evaluación neuropsicológica en individuos con CET cobra un carácter importante al proporcionar información sobre los déficits cognitivos específicos que subyacen la afectación cerebral, y que alteran el funcionamiento intelectual y sobre aspectos de sus comportamientos adaptativos. Asimismo, el estudio del perfil cognitivo permite observar el nivel de funcionamiento del individuo y sus necesidades de apoyo, determinando qué áreas están más preservadas y cuáles presentan mayor compromiso. Es de vital importancia este último para la planificación de la intervención neuropsicológica [10]. Toda exploración neuropsicológica en el CET debe considerar aspectos como los antecedentes clínicos y el ambiente del paciente, ya que existen mecanismos neurobiológicos diferentes en este tipo de población que dan lugar a fenotipos cognitivos específicos y variables. La planificación de la intervención neuropsicológica debe tener en cuenta dichos elementos y precisar lo que más pueda un perfil semiológico adecuado [10].

Según Vries y Veltman [11], en aquellos casos donde el paciente no tiene DI, por lo general no se presenta un déficit más prominente en las habilidades atencionales, funciones ejecutivas y habilidades visoespaciales. Asimismo, en el estudio de Sharma y Rao [12] se descartó DI en su paciente; sin embargo, en la evaluación neuropsicológica, el paciente presentó más compromiso en dominios cognitivos como la atención, concentración, memoria de trabajo, planificación, capacidad integradora y recuerdo, con presencia de confabulaciones.

Aquellos individuos con diagnóstico de CET y comorbilidad con DI leve a moderada presentan algunos dominios preservados y otros más afectados. Los dominios típicamente más preservados son la memoria a corto plazo de tipo verbal, el razonamiento no verbal y lenguaje receptivo [13], mientras que, las habilidades psicomotoras, la velocidad de procesamiento, la memoria de trabajo (especialmente la visoespacial), la memoria fonológica, el lenguaje expresivo, función ejecutiva y habilidades adaptativas se hallan frecuentemente con mayor grado de afectación [5]. En la etapa infantil muestran claro retraso en la adquisición de funciones comunicativas (para iniciar y mantener una conversación), lo que puede deberse al retraso en el desarrollo léxico y fonológico, durante el cual el aprendizaje de palabras es más lento que el de un niño con desarrollo típico. A pesar de esto, es posible que estas dificultades mejoren al final de la infancia y adolescencia; no obstante, depende de la gravedad de la afectación [5].

Uno de los procesos cognitivos que al parecer presenta más afectación en esta patología es el de las funciones ejecutivas, posiblemente por la gran influencia que tiene en la conducta adaptativa, el rendimiento académico y el correcto funcionamiento de otros procesos cognitivos. En términos generales, el funcionamiento ejecutivo es la capacidad que tiene el ser humano para planificar, organizar, inhibir, monitorizar la conducta y ser flexible ante situaciones novedosas [14]. Respecto a la evaluación de la función ejecutiva en población con CET y DI, es de resaltar que la mayoría de los estudios evalúan aspectos específicos como la atención y la memoria de trabajo. Quizás sea porque en la actualidad no se cuenta con suficientes instrumentos neuropsicológicos adecuados para esta población, acentuados, específicamente, en la función ejecutiva [8].

Según Danielsson, Messer y Rönnberg [15], se realizó una investigación sobre función ejecutiva en población adulta con DI durante un periodo de 5 años con una muestra conformada por 46 adultos con DI y un grupo control conformado por 92 adultos sin DI. La población 
de estudio era perteneciente a la ciudad de Umea (norte de Suecia) y el rango de edad oscilaba entre los 40 y 85 años. Los instrumentos escogidos en la evaluación de las funciones ejecutivas fueron la Torre de Hanói, fluidez verbal y memoria de palabra. Los resultados arrojaron que las personas con DI presentaban más alteraciones en la fluidez verbal y en la memoria de trabajo y no se hallaron diferencias significativas en la planificación, ya que el número de movimientos utilizados en la Torre de Hanói fue similar al del grupo control. Sin embargo, esto se explica porque la Torre de Hanói no solo evalúa planeación, sino también la memoria de trabajo (información visoespacial), la actualización y la flexibilidad cognitiva. Para Danielsson et al. [5-15], las principales fallas se presentan en procesos como la inhibición de respuestas, la planificación y la memoria de trabajo no verbal, con un mejor desempeño en procesos como la fluidez y el cambio de foco atencional. Frente a esto, otros autores convergen al indicar que se observan deficiencias en procesos como la inhibición y planificación ante pruebas como la Torre de Hanói y laberintos [8-15] en contraste con el desempeño obtenido ante pruebas que evalúan procesos de atención focal, sostenida, y memoria a corto plazo verbal. En consecuencia, estas dificultades en el funcionamiento ejecutivo se pueden asociar con el fracaso escolar, social y personal que experimentan la mayoría de los individuos con DI en su vida cotidiana.

El uso de las nuevas tecnologías se implementa cada vez más en rehabilitación neuropsicológica ya que intenta entrenar la atención, memoria de trabajo y funciones ejecutivas, destacando los siguientes componentes: graduar la complejidad de la tarea; dividir las tareas según los componentes; impartir instrucciones claras y simples; utilizar recursos que sean asequibles; plantear actividades que sean ecológicas para el individuo (generalización del aprendizaje e intervención ecológica) y, por último, fomentar estrategias de automonitoreo en el individuo con DI de leve a moderada [16]. De acuerdo con Cárdenas, Rojas y Cuellar [17], se realizó un estudio que tenía como objetivo evaluar la eficacia de un protocolo de estimulación cognitiva enfocado en el fortalecimiento de la atención en pacientes con diagnóstico de DI. La muestra estuvo conformada por 12 adultos pertenecientes a la Fundación para el Desarrollo Integral del Niño Especial (FUNDINES), con edades entre los 29 y 54 años. Se discutieron dos aspectos: el primero, la pertinencia de una rehabilitación enfocada en una habilidad cognitiva especifica (atención); el segundo, el número de sesiones requeridas para la consolidación del aprendizaje. Como resultado, se obtuvieron cambios positivos en el funcionamiento cognitivo general que fueron corroborados por la prueba de tamizaje MoCA. Asimismo, Torres, Galvis, Lopera, y Montoya [18] desarrollaron un estudio con una muestra poblacional de 20 niños y niñas de la región de Antioquia, Colombia, con DI entre los 7 y 12 años. El grupo caso tuvo un proceso de entrenamiento con el programa computarizado Lumosity. Después de la intervención se comprobó la eficacia del dispositivo tecnológico al observar un cambio frente a un antes y un después en el funcionamiento ejecutivo (planeación y flexibilidad).

Finalmente, estas investigaciones aportan información útil sobre el perfil neuropsicológico en población con CET con comorbilidad en DI; sin embargo, siguen siendo pocos los estudios cuyo objetivo está orientado hacia conocer el perfil cognitivo en este tipo de población y su posible rehabilitación. Es preciso resaltar que la intervención neuropsicológica en pacientes con CET y DI tendría un efecto en el funcionamiento cognitivo.

\section{Metodología}

Para la realización de esta investigación se utilizó un diseño de estudio de caso para Martínez [19] que, básicamente, se trata de una estrategia metodológica útil en la generación de resultados que posibilitan el crecimiento, desarrollo y fortalecimiento de las teorías existentes o el surgimiento de nuevos paradigmas científicos. Dicha investigación se desarrolló desde el enfoque empírico analítico del caso de un adulto con Complejo de Esclerosis Tuberosa, epilepsia y 
Discapacidad Intelectual. Este tipo de diseño, siguiendo a Muñoz, Quintero, y Munévar [20], tiene como característica básica explicar, predecir y controlar los fenómenos experimentables, valiéndose de la observación y medición, y, dentro de estas, considerando la cuantificación como una característica directa de la medición.

La investigación se llevó a cabo a través de un proceso de evaluación neuropsicológica enfocada en mostrar el perfil cognitivo de una paciente de 25 años diagnosticada desde los 7 años con CET, epilepsia y DI. Para esto se utilizó la observación clínica, una entrevista semiestructurada con docentes, auxiliares y paciente, la elaboración de historia clínica y aplicación e interpretación de diferentes pruebas neuropsicológicas. A partir de ahí, se compararon los resultados encontrados con lo reportado en la literatura; se realizó una descripción de las mediciones y se planteó una intervención neuropsicológica de la función ejecutiva dorsolateral, que mejorará el desempeño de actividades instrumentales en la paciente.

\section{Caso clínico}

Adulta femenina de 25 años, en medida de restauración de derechos por parte del ICBF bajo la modalidad de hogar sustituto en el Instituto de Capacitación los Álamos, es remitida a neuropsicología para valoración del Coeficiente Intelectual y funcionamiento cognitivo actual.

La paciente presenta antecedentes posnatales importantes como desnutrición y maltrato infantil. A la edad de 6 años ingresa a medida de protección y restauración de derechos por parte del ICBF. A los 7 años es diagnosticada con CET al presentar máculas hipocrómicas en tronco y miembros, con pápulas cupuliformes eritematosas en región cefálica. Posteriormente es medicada con media tableta de $0.5 \mathrm{mg}$ de Rivotril cada 12 horas para el control de convulsiones. Tuvo una intervención quirúrgica en el 2019 para retiro de angiomiolipoma renal. Tiene pendiente una Resonancia Magnética Nuclear (RMN) para determinar el tipo de lesiones cortical que presenta. Tiene antecedentes de dificultades en el aprendizaje y estudios de primaria incompletos.

En la actualidad, la docente y auxiliar de B refieren:

Es muy lenta para aprender. En la mayoría de las ocasiones se le dificulta llevar un orden en las tareas, se evidencia pobre planeación y secuenciación en actividades. La usuaria es dependiente en actividades como preparación de alimentos, manejo de dinero, pago de recibos, etc. Es necesario que ella adquiera estas competencias para poder acceder a la modalidad de vivienda en comunidad, una modalidad del Instituto Álamos que le permitiría ser más independiente.

$\mathrm{Al}$ estar en medida de protección y restauración de derechos, se elaboró, por parte de los investigadores, un consentimiento informado con el fin de obtener la aprobación de ICBF para la realización del estudio de caso, aclarando los objetivos de la investigación, la importancia de esta para la construcción del cuerpo teórico y su posterior presentación en la comunidad académica. Dicho consentimiento también fue presentado y avalado por el Comité de Ética de la Universidad de San Buenaventura, sede Medellín.

Para el proceso de evaluación neuropsicológica se utilizó el protocolo del Instituto Álamos y algunas pruebas sugeridas en la guía de DI con validación en poblaciones similares: Escala de Inteligencia Wechsler (WAIS IV); Inventario para la Planificación de Servicios y Programación Individual ICAP Bruinin de [21]; test de copia de una figura compleja (figura de Rey) [22]; prueba de dígitos directos e indirectos y letras y números del WAIS-III [23]; el test de cancelación de letras; Trail Making Test (TMT); escala de memoria de Wechsler [24]; token test [25]; test de vocabulario de Boston-BNT [26]; y la Batería Neuropsicológica de Funciones Ejecutivas y Lóbulos Frontales (BANFE) (manual moderno). Una vez realizada la evaluación del coeficiente intelectual a través del WAIS IV y el ICAP, se procedió 
a evaluar cada uno de los procesos cognitivos de B (gnosias, praxias, atención, memoria, lenguaje y funcionamiento ejecutivo).

\section{Resultados}

Las pruebas neuropsicológicas empleadas en este estudio quedan reflejadas en la Tabla 1. Para la administración y valoración de dichas pruebas, se siguieron las normas indicadas según los manuales. Durante la evaluación neuropsicológica, B se mostró colaboradora, atenta, orientada en tiempo, espacio y persona. En la ejecución de las actividades se mantuvo con actitud dispuesta de principio a fin, cumpliendo a cabalidad con las tareas. Su lenguaje estuvo poco fluido y prosódico; mantuvo reciprocidad en la comunicación, aunque con dificultad a nivel pragmático. Como resultado de la evaluación del Coeficiente Intelectual (CI), B obtuvo un CI Total de 65 en la Escala de Inteligencia Wechsler (WAIS IV), lo cual la ubica dentro de un rango muy bajo para su edad, con perfil homogéneo sin discrepancias significativas. Si bien su desempeño está por debajo de su edad y escolaridad, evidencia, de forma sutil, una mejor competencia en razonamiento perceptual.

A nivel de funcionamiento, la paciente se ubica dentro del rango de limitada atención a la persona y/o seguimiento periódico, según el puntaje obtenido en la prueba de Escala Funcional y Comportamental (ICAP). Tiene adecuadas destrezas motoras, sociales y comunicativas, pero presenta ligeras deficiencias en la autonomía y en la planificación de actividades para satisfacer sus propias necesidades en el marco del hogar. La paciente muestra dificultades para responder adecuadamente a los requerimientos económicos y sociales del mundo laboral y de otras situaciones sociales; sin embargo, son esperables dada su situación actual. Si bien presenta problemas de conducta en lo que concierne a daños a otros a través del golpe y la mordida, la gravedad de estos eventos es un problema moderado, pues tienen una frecuencia de una a tres veces al mes.

Con respecto a las actividades que evalúan gnosias, B presenta dificultades en las habilidades perceptuales para el análisis y reconocimiento de los aspectos esenciales de un objeto, formación de conceptos no verbales, razonamiento de analogías visuales y seriales e integración de información visual (ver Figura 1).

De acuerdo con lo observado en la ejecución de tareas, se evidencian dificultades para la síntesis de organización y planeación visoespacial, coordinación visomotora y construcción de objetos tridimensionales (ver Figura 2).

Durante la evaluación, se evidenció que B tiene capacidades básicas para sostener su atención durante una tarea y seleccionar estímulos con el fin de cumplir una meta, con fallas en la alternancia atencional que dificultan procesos de atención compleja y mantenimiento de foco ante tareas que le implican mayor esfuerzo cognitivo. Presenta deficiencias en la velocidad de procesamiento que comprometen su capacidad para focalizar, explorar y ordenar con rapidez y eficacia (ver Figura 3). 


\begin{tabular}{|c|c|c|c|c|}
\hline Función Cognitiva & Prueba & P. Natural & P. Escalar & Valor normal \\
\hline \multirow{4}{*}{ Gnosias } & Razonamiento perceptual WAIS IV & & & \\
\hline & Diseño con cubos & 20 & 4 & $(\mathrm{~N}: 10 \pm 3)$ \\
\hline & Matrices & 13 & 7 & $(\mathrm{~N}: 10 \pm 3)$ \\
\hline & Rompecabezas visual & 7 & 4 & $(\mathrm{~N}: 10 \pm 3)$ \\
\hline \multirow{3}{*}{ Práxias } & Gubos de WAIS & 20 & 4 & $(\mathrm{~N}: 10 \pm 3)$ \\
\hline & Copia figura compleja de REY (Puntuación) & 18 & & $32.51 \pm 4.18$ \\
\hline & Tiempo* & $150 "$ & & $157.01 \pm 85.98$ \\
\hline \multirow{17}{*}{ Atención } & Ejecución continua & & & \\
\hline & Visual: & 16 & & $>14$ \\
\hline & Tiempo* & $37^{*}$ & & \\
\hline & Errores & 0 & & \\
\hline & Auditiva & 14 & & $>14$ \\
\hline & Tiempo* & $60^{*}$ & & \\
\hline & Errores & 3 & & \\
\hline & TMT A & & & \\
\hline & Aciertos & 24 & & \\
\hline & Errores & 1 & & \\
\hline & Tiempo* & $219^{\prime \prime}$ & & $107,53^{\prime \prime}$ \\
\hline & Dígitos WEGHSLER & 9 & 4 & $(\mathrm{~N}: 10 \pm 3)$ \\
\hline & Orden directo & 4 & & \\
\hline & Regresión & 5 & & \\
\hline & Glaves: digito-símbolo WAIS IV & 4 & & $(\mathrm{~N}: 10 \pm 3)$ \\
\hline & Volumen de aprehensión de información & & & \\
\hline & Span Verbal & 4 & 5 & $(\mathrm{~N}: 10 \pm 3)$ \\
\hline \multirow{28}{*}{ Memoria } & Memoria de Wechsler & & & \\
\hline & Presentación Auditiva & & & \\
\hline & Textos & & & \\
\hline & TIRe Recuerdo de unidades A + B & 18 & 2 & $(\mathrm{~N}: 10 \pm 3)$ \\
\hline & Textos II & & & \\
\hline & TIIRe Recuerdo unidades A+B & 16 & 5 & $(\mathrm{~N}: 10 \pm 3)$ \\
\hline & Reconocimiento & 19 & & $(\mathrm{~N}: 10 \pm 3)$ \\
\hline & Lista de Palabras I & & & \\
\hline & LPIA1 Recuerdo primer intento & 4 & 5 & $(\mathrm{~N}: 10 \pm 3)$ \\
\hline & LPIRe Recuerdo (+ de los 4 ensayos) & 24 & 2 & $(\mathrm{~N}: 10 \pm 3)$ \\
\hline & Lista de Palabras II & & & \\
\hline & LPIIA Lista A & & & \\
\hline & LPIIRe Recuerdo & 7 & 7 & $(\mathrm{~N}: 10 \pm 3)$ \\
\hline & LPIIRec Reconocimiento & 21 & 5 & $(\mathrm{~N}: 10 \pm 3)$ \\
\hline & LPIIPr Retención & 77 & 7 & $(\mathrm{~N}: 10 \pm 3)$ \\
\hline & Presentación visual & & & \\
\hline & Escenas I & & & \\
\hline & EsIRe Recuerdo & 30 & 5 & $(\mathrm{~N}: 10 \pm 3)$ \\
\hline & Escenas II & & & \\
\hline & EsIIRe Recuerdo & 23 & 4 & $(\mathrm{~N}: 10 \pm 3)$ \\
\hline & EsPR porcentaje de retención & 76 & 3 & $(\mathrm{~N}: 10 \pm 3)$ \\
\hline & Evocación Figura compleja & & & \\
\hline & Recuerdo inmediato & & & \\
\hline & Puntuación memoria & 6 & 11,42 & 7,75 \\
\hline & Tiempo* & $70 "$ & $224,31 ”$ & $135,37 ”$ \\
\hline & Memoria de trabajo WAIS IV & 55 & & $(\mathrm{~N}: 100 \pm 15)$ \\
\hline & Retención de dígitos & 12 & 2 & $(\mathrm{~N}: 10 \pm 3)$ \\
\hline & Aritmética & 5 & 3 & $(\mathrm{~N}: 10 \pm 3)$ \\
\hline \multirow{14}{*}{ Lenguaje } & Comprensión verbal WAIS IV & 31 & & $(\mathrm{~N}: 100 \pm 15)$ \\
\hline & Token Test & 127 & & 161 \\
\hline & TEST DE BOSTON & & & \\
\hline & Vocabulario & 36 & & 43,92 \\
\hline & Correcta sin clave & 23 & & \\
\hline & Correcta con clave semántica & 1 & & \\
\hline & Correcta con clave fonológica & 5 & & \\
\hline & Fluidez & & & \\
\hline & Fluidez semántica & & & \\
\hline & Animales & 15 & & $18.38 \pm 4.09$ \\
\hline & Frutas & 10 & & \\
\hline & Promedio & 12,5 & & \\
\hline & Perdida de categoría & 0 & & \\
\hline & Perseveraciones & 9 & & \\
\hline \multirow{6}{*}{ Fx Ejecutiva } & BANFE & & & \\
\hline & Puntuaciones totales & P. Natural & P. Normal & Diagnóstico \\
\hline & Subtotal orbitomedial & 145 & & Alteración severa \\
\hline & Subtotal prefrontal anterior & 9 & 42 & Alteración severa \\
\hline & Total, dorsolateral $(\mathrm{MT}+\mathrm{FE})$ & 124 & 63 & Alteración severa \\
\hline & Total, Batería de Funciones Ejecutivas & 278 & & Alteración severa \\
\hline
\end{tabular}

* El tiempo se presenta en segundos (") 


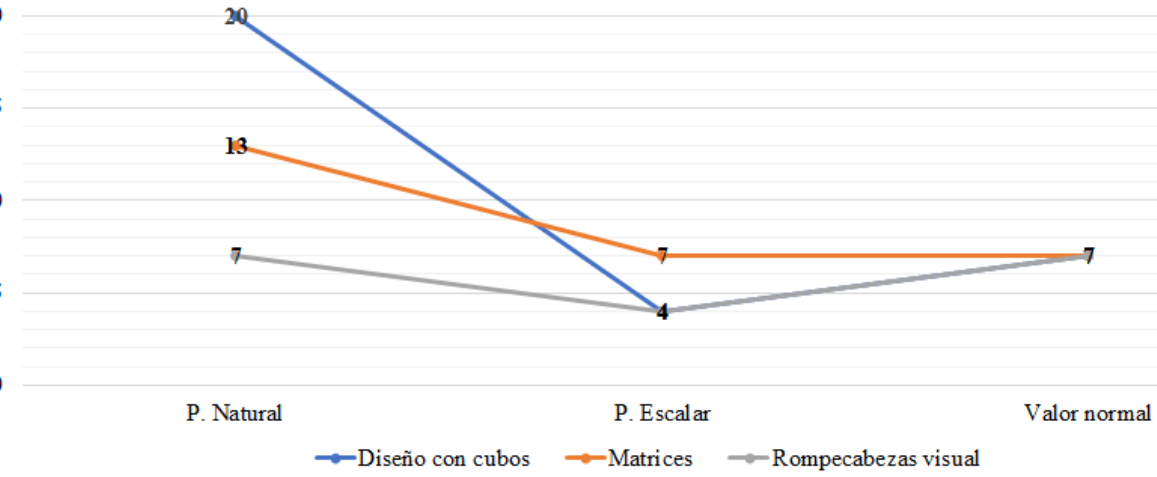

Figura 1: evaluación de procesos gnósicos.

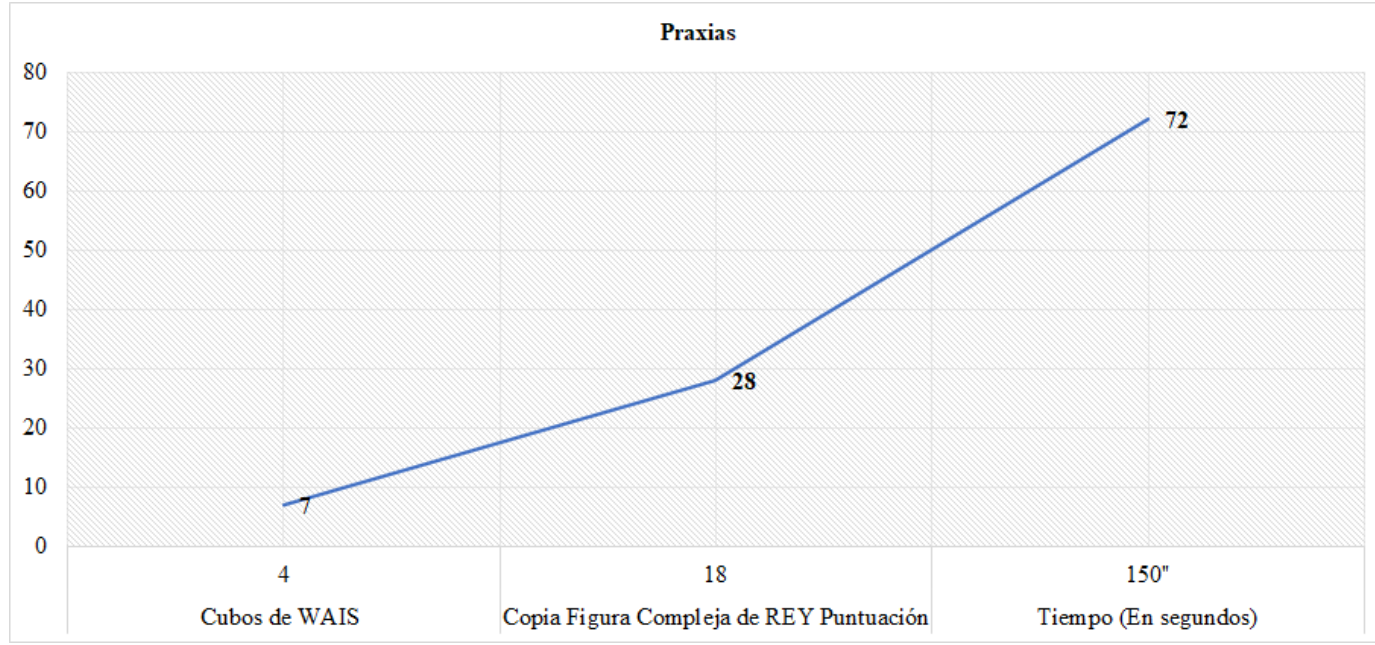

Figura 2: evaluación de praxias

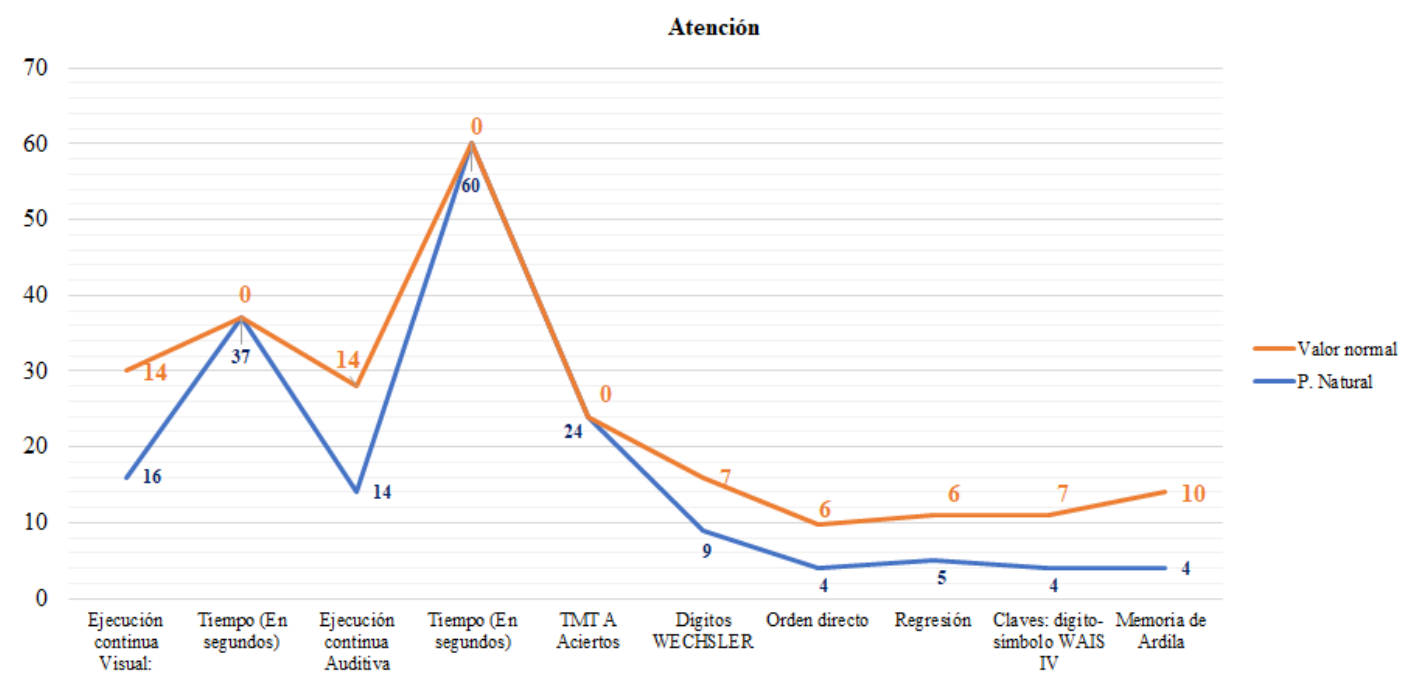

Figura 3: evaluación de procesos atencionales 
La paciente muestra pocas capacidades básicas de registro, almacenamiento y evocación de la información con fallas en el sistema de retención y almacenamiento de estímulos auditivos y visuales de la memoria inmediata. Presenta dificultad variable para coordinar, de manera simultánea, varias actividades que requieran la manipulación de la información, específicamente en la transformación de la información visual a la verbal, con afectaciones significativas en lo que concierne a la memoria operativa. Frente a tareas de reconocimiento no hay un beneficio significativo. Aunque existe un grado de variabilidad en el desempeño de las pruebas, se observa que a la paciente se le facilita más el proceso de aprendizaje y memoria visual con apoyo de tareas de ejecución (ver Figura 4 y 5).

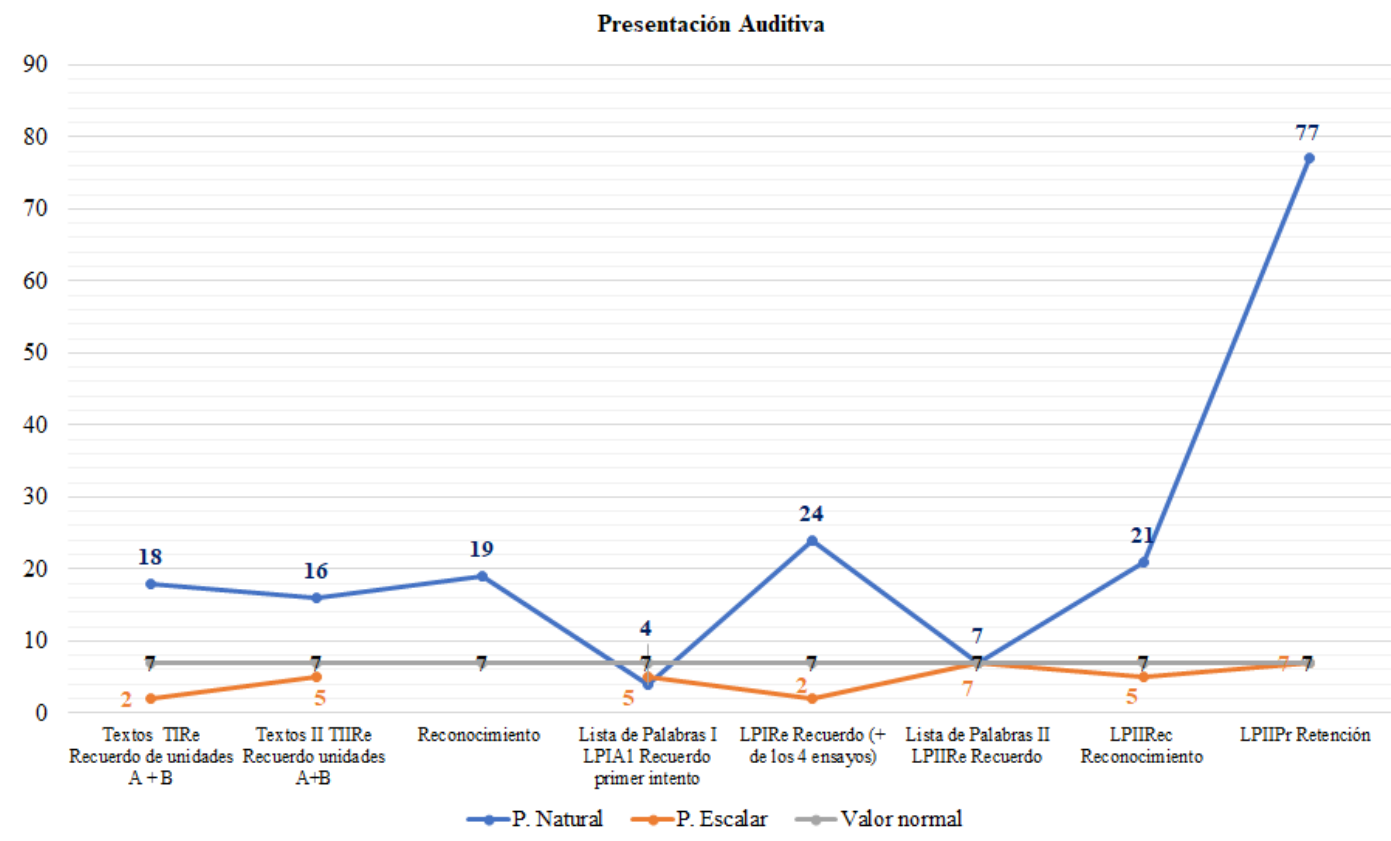

Figura 4: evaluación de procesos mnésicos - presentación auditiva

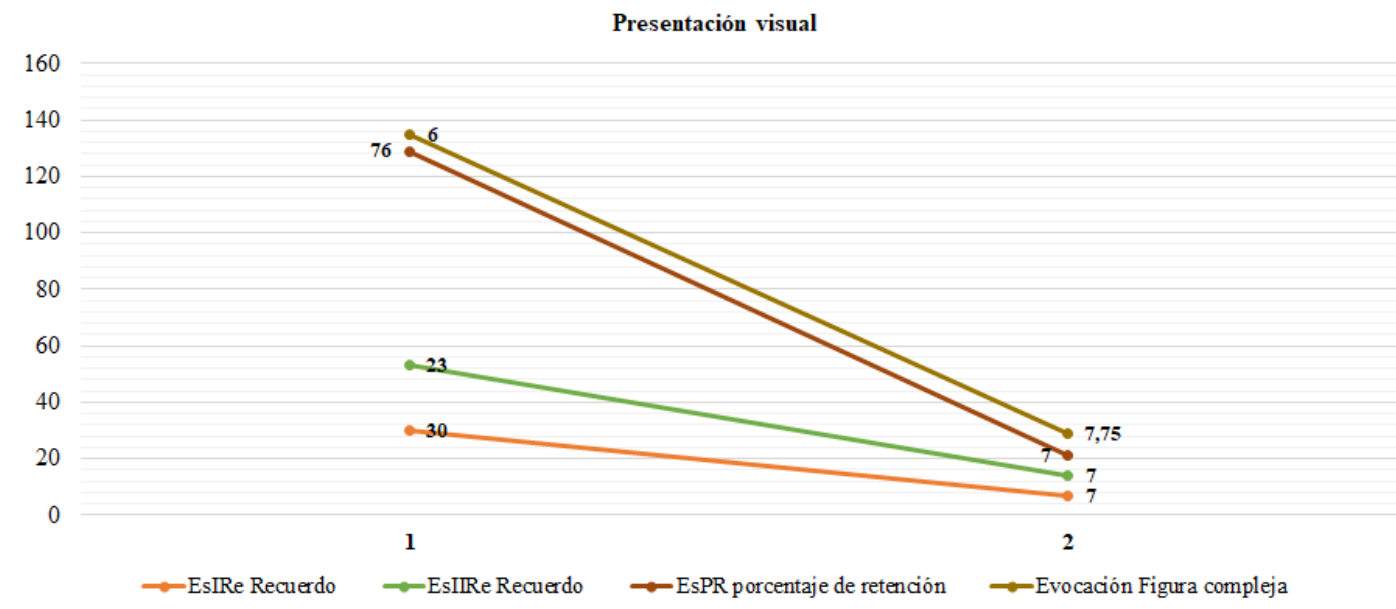

Figura 5: evaluación de procesos mnésicos - presentación visual 
A nivel expresivo, se evidencia anomia con pocas estrategias para iniciar la comunicación verbal oral, fallas en la fluidez verbal y en la capacidad de repetición. A nivel receptivo, presenta fallas para dotar de significado el lenguaje oral así como de la integración de los estímulos visuales y auditivos que permiten la comprensión del lenguaje. B presenta dificultades en formación de conceptos verbales, expresión de la relación entre conceptos; riqueza y precisión en la definición de vocablos; fallas en el razonamiento y juicio social frente a la solución de problemas cotidianos, lo que es esperable dado que la paciente proviene de un entorno privativo que le brinda pocas oportunidades para desarrollar aspectos de conocimiento sociocultural (ver Figura 6).

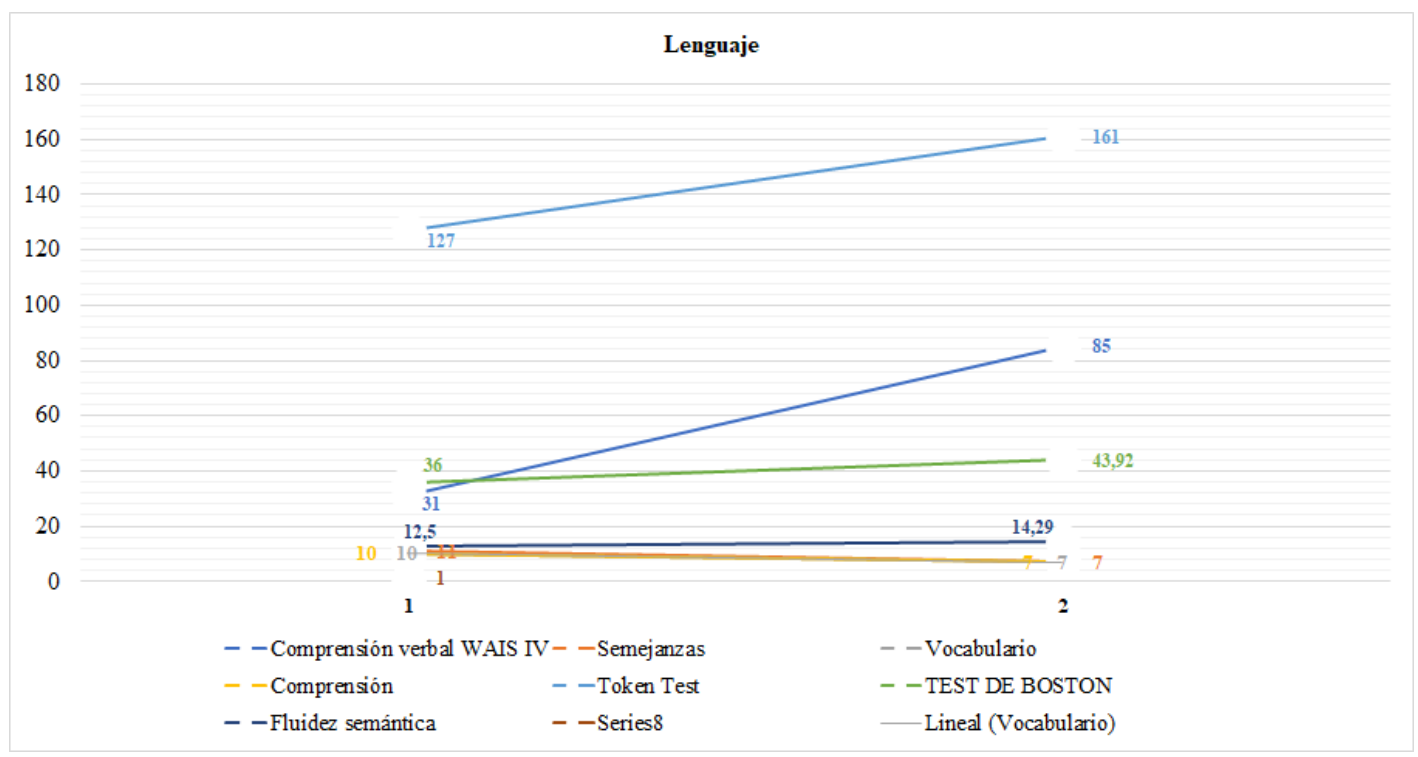

Figura 6: evaluación del lenguaje

La paciente presenta fallas en la planificación, organización y ejecución de tareas necesarias para realizar una operación cognitiva a través de la manipulación de información verbal y visual (MT). No logra realizar actividades que requieran de la planeación secuencial para resolver problemas a través de acciones que solo juntas y en sucesión conllevan a una meta específica. No selecciona esquemas de acción para resolver un problema que requiere movimientos contraintuitivos y ordenarlos de manera progresiva. A pesar de que los resultados de la evaluación indiquen alteración severa (lo cual es compatible con la literatura), se observa que la paciente posee adecuado control inhibitorio de respuestas inadecuadas y que regula su comportamiento por medio de autoinstrucciones. También tiene habilidades para almacenar de manera temporal (MT) información de tipo visual para alcanzar objetivos inmediatos. Además, posee adecuada capacidad para generar criterios de clasificación, pero, sobre todo, capacidad para modificarlos (flexibilidad) (ver Figura 7). 


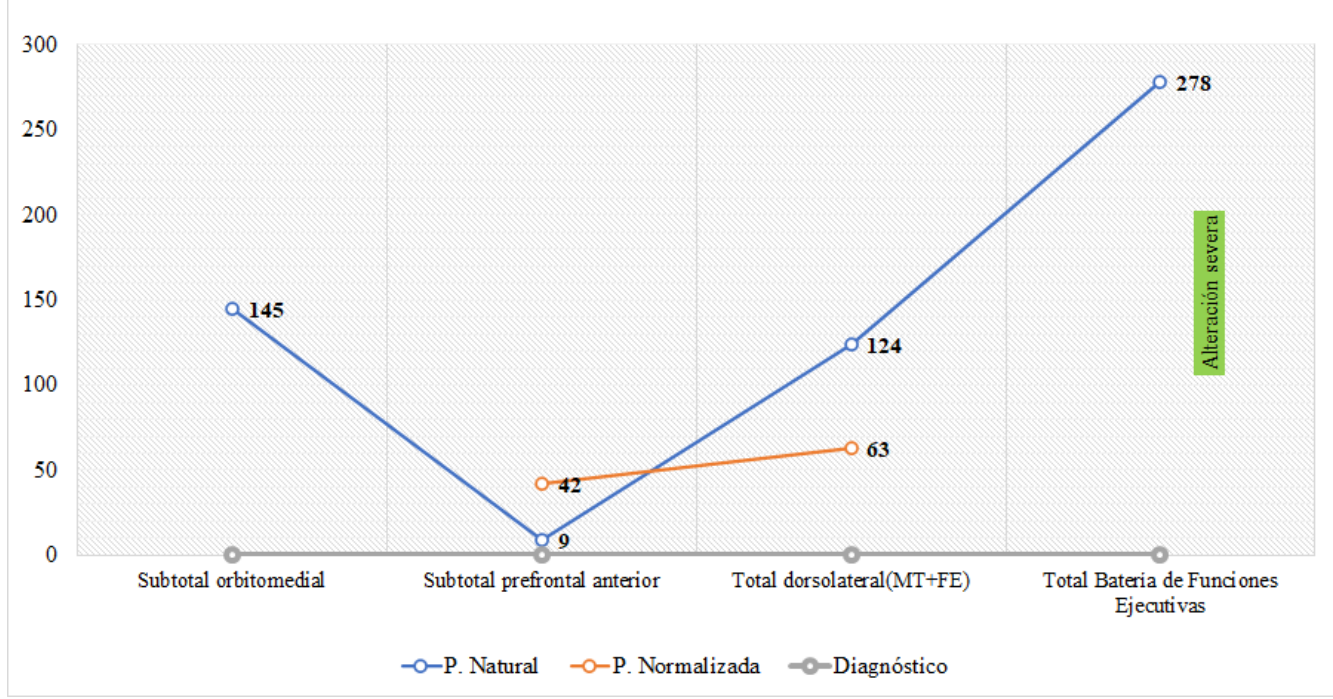

Figura 7: evaluación de funcionamiento ejecutivo

Se realizó una medición de las tareas del área dorsolateral de la batería BANFE con el fin de valorar, a profundidad, el desempeño de B ante actividades que requieren planeación, abstracción, memoria de trabajo, seriación, secuenciación y flexibilidad mental. Asimismo, se utilizó el cuestionario neuropsicológico de daño frontal de la misma batería (aplicado a la docente de B). Los resultados obtenidos en cuatro momentos diferentes permitieron conocer cómo la red de apoyo de la paciente percibe su grado de autoconciencia, sus intereses, motivaciones, el control conductual, su personalidad, entre otras variables que inciden en su funcionamiento. Como resultado de las cuatro mediciones, se obtuvo en ambas pruebas un patrón de respuesta y conducta estable, con un puntaje natural mínimo de 120 y puntaje natural máximo de 128 para las tareas del área dorsolateral, que indican que B presenta severas dificultades en el funcionamiento de dicha área (ver Figura 8).

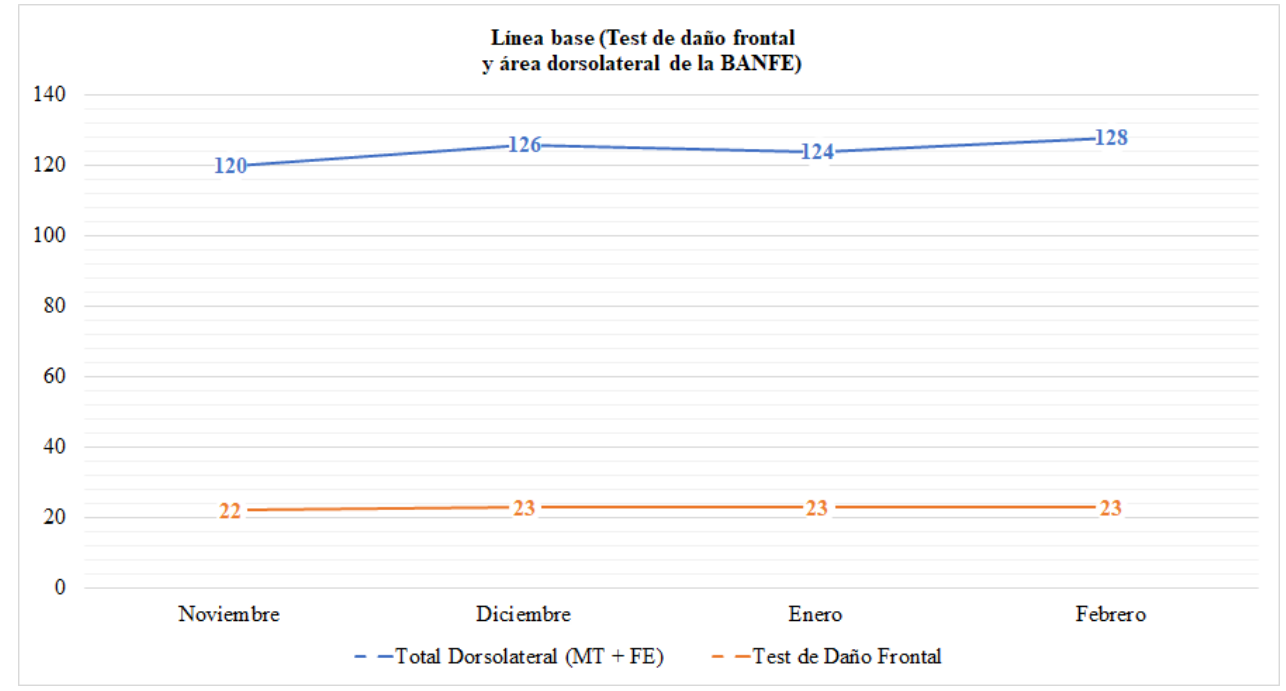

Figura 8: mediciones del funcionamiento ejecutivo dorsolateral e inventario del daño frontal 


\section{Propuesta de intervención neuropsicológica}

El programa de intervención se desarrolló a partir de tres modelos, los cuales concordaban con la valoración inicial y las necesidades de la paciente y su contexto: el Modelo Multidimensional del Funcionamiento Humano [27], donde la presencia de DI implica la interacción dinámica y recíproca de múltiples factores como el funcionamiento intelectual, las habilidades adaptativas, la salud, la participación y el contexto, bajo la premisa de que si se brindan apoyos individualizados acorde a sus necesidades las personas pueden mejorar su funcionamiento; el Modelo Teórico de las Funciones Ejecutivas [28] que define conceptualmente el funcionamiento ejecutivo dorsolateral y sus procesos; y, por último, el Modelo de Rehabilitación de Funcionamiento Ejecutivo de [29], que se fundamenta en tres fases: la selección y ejecución de tareas cognitivas, manejo apropiado del tiempo y el establecimiento de estrategias que beneficien la ejecución de planes (automonitoreo).

El proceso de intervención se plantea a partir de estas tres etapas con sus respectivos objetivos específicos y sustentadas en estrategias de estimulación y compensación del funcionamiento ejecutivo dorsolateral. Se proponen dos sesiones semanales, con una duración de 60 minutos, para un total de 16 sesiones, las cuales están diseñadas de acuerdo con las etapas anteriormente descritas. Las áreas a trabajar y objetivos se exponen en la Tabla 2:

Tabla 2. Propuesta de rehabilitación neuropsicológica

\begin{tabular}{|c|c|c|}
\hline Sesión & Objetivo específico & Área trabajada \\
\hline 1 & Explicar proceso de rehabilitación y actualizar estado de la paciente. & Consulta de ingreso a rehabilitación y encuadre sobre el proceso. \\
\hline 2 & \multirow{3}{*}{$\begin{array}{l}\text { Implementar mecanismo de estimulación en procesos atencionales } \\
\text { tanto simples como complejos. }\end{array}$} & Atención sostenida, selectiva y alternante. \\
\hline 3 & & Memoria de Trabajo o Atención Compleja \\
\hline 4 & & Velocidad de Procesamiento de la Información \\
\hline 5 & \multirow{3}{*}{$\begin{array}{l}\text { Incentivar la capacidad para formular varias soluciones o hipótesis } \\
\text { ante diferentes situaciones, estructurar planes de acción y elegir el más } \\
\text { apropiado. }\end{array}$} & Fx Ejecutiva: generación de hipótesis \\
\hline 6 & & Fx Ejecutiva: generación de hipótesis \\
\hline 7 & & Fx Ejecutiva: generación de hipótesis \\
\hline 8 & $\begin{array}{l}\text { Estimular procesos ejecutivos como planeación y secuenciación, con el } \\
\text { fin de lograr mejoras en actividades que impliquen la organización de } \\
\text { las tareas del hogar y su rutina diaria. }\end{array}$ & Fx Ejecutiva: planeación y secuenciación \\
\hline 9 & $\begin{array}{l}\text { Reforzar procesos de funciones ejecutivas con el fin de potencializar en } \\
\text { la paciente la capacidad de calcular, estructurar, pensar e idear un plan } \\
\text { que le permita llevar a cabo una tarea. }\end{array}$ & Fx Ejecutiva: planeación y secuenciación \\
\hline 10 & $\begin{array}{l}\text { Fortalecer procesos de funciones ejecutivas con el fin de potencializar } \\
\text { en la paciente la capacidad de idear una mejor alternativa a la hora de } \\
\text { planear una actividad, permitiéndole realizarla de manera efectiva. }\end{array}$ & Fx Ejecutiva: planeación \\
\hline 11 & $\begin{array}{l}\text { Estimular procesos ejecutivos de seriación, con el fin de lograr mejoras en } \\
\text { actividades que requieran la preparación de alimentos. }\end{array}$ & Fx Ejecutiva: seriación \\
\hline 12 & $\begin{array}{l}\text { Promover en la paciente procesos de eficiencia y manejo del tiempo a } \\
\text { partir de rutinas alimentarias. }\end{array}$ & Fx Ejecutiva: eficiencia y manejo del tiempo \\
\hline 13 & $\begin{array}{l}\text { Implementar estrategias de entrenamiento que le permitan a la paciente } \\
\text { realizar o cumplir adecuadamente una tarea y ganarle al reloj. }\end{array}$ & Fx Ejecutiva: eficiencia y manejo del tiempo \\
\hline 14 & $\begin{array}{l}\text { Implementar estrategias de compensación con el fin de que sean } \\
\text { implementadas en la vida cotidiana. }\end{array}$ & $\begin{array}{l}\text { Funcionalidad en actividades instrumentales (tomarse los } \\
\text { medicamentos). }\end{array}$ \\
\hline 15 & $\begin{array}{l}\text { Implementar estrategias de compensación con el fin de que se genere } \\
\text { mayor autonomía y permita que la paciente haga uso de las estrategias } \\
\text { de automonitoreo. }\end{array}$ & Funcionalidad en actividades instrumentales (realizar compras). \\
\hline 16 & Cierre del proceso & $\begin{array}{l}\text { Consulta de egreso. Plan casero, devoluciones a la paciente y } \\
\text { su red de apoyo. }\end{array}$ \\
\hline
\end{tabular}


Con este protocolo de rehabilitación se espera mejorar el funcionamiento cotidiano de la paciente a través de la recuperación del funcionamiento ejecutivo, que según las investigaciones se relaciona directamente con las habilidades adaptativas, la productividad y la participación en su contexto natural. En primer lugar, se espera estimular los procesos atencionales tanto simples como complejos, entendiendo que son la base para acciones cognitivas más elaboradas. A continuación, el tratamiento se enfocará en estimular la memoria de trabajo y la velocidad de procesamiento con el fin de afianzar procesos de razonamiento y aprendizaje. Finalmente, se centrará en que la paciente logre estimular y entrenar procesos como la planeación, organización, generación de hipótesis, eficiencia y manejo del tiempo, logrando así mejorar competencias y habilidades que repercutirán positivamente en su nivel de independencia cotidiana. La propuesta completa con el diseño de las actividades ha quedado en el Instituto de Capacitación los Álamos para ser implementada.

\section{Discusión}

Las conclusiones de la evaluación neuropsicológica permitieron corroborar la prevalencia de DI en el CET al observar que los resultados de la evaluación del Coeficiente Intelectual de B están por debajo de lo esperado para su edad (con un CI Total de 65), y que su desempeño en actividades instrumentales está igualmente por debajo, lo que la hace una persona con necesidad de atención y seguimiento periódico. Lo anterior linda con los estudios de Ruggieri y Arberas [30] quienes refieren que los pacientes con CET tienen un rendimiento variable en la evaluación neuropsicológica desde el punto de vista intelectual; sin embargo, pueden estar más predispuestos a presentar DI aquellos con lesiones corticales por tubérculos ubicadas en los lóbulos frontales. Asimismo, autores como Sorokin, Remes, Lenicov, y Pereira [31] postulan que los hamartomas de la sustancia gris se ubican predominantemente en los lóbulos frontales, y su tamaño y cantidad empeoran la disfunción cerebral asociada a la epilepsia refractaria y llevan el deterioro cognitivo de moderado a grave. En esta misma línea, los estudios sobre el deterioro cognitivo a causa de las convulsiones por parte de los autores Gómez y Vargas [32] sugieren que las crisis más frecuentes son focales y pueden deberse a lesiones de quistes aracnoideos. Aquellas crisis con peor pronóstico a nivel cognitivo y comportamental son las de inicio precoz, como los espasmos infantiles y descargas epileptiformes en el lóbulo temporal. Lo anterior coincide con la situación de la paciente, ya que esta presentó dichos síntomas y en la actualidad tiene diagnóstico de epilepsia.

La evaluación permitió dilucidar dificultades en las habilidades perceptuales, lo que concuerda con lo postulado por Sorokin et al. [33] que refieren que las tuberosidades pueden alojarse en el lóbulo parietal, comprometiendo así la región occipito-dorsolateralparietal e incidiendo en las habilidades perceptuales apercetivas y asociativas. Asimismo, presentó dificultades en tareas que requerían velocidad de procesamiento, otro aspecto en el cual se puede especular, ya que los estudios de García, Esteba, y Viñas [13] sugieren que la velocidad de procesamiento puede estar comprometida por lesiones subcorticales y sus respectivas conexiones. Igualmente, los estudios de Gómez y Vargas [32] proponen que al existir lesiones en el SNG a causa de astrocitoma subependimario de células gigantes (SEGA), estas se localizarían junto a la cabeza del núcleo caudado, contribuyendo a dificultades con sus múltiples conexiones en lo que refiere al control de movimiento, memoria y aprendizaje. De igual manera, B presentó fallas significativas en el proceso de retención y almacenamiento de información visual y auditiva de memoria inmediata y a largo plazo, con afectación significativa en la memoria de trabajo más de tipo visual y dificultades en la formación de conceptos verbales, en la expresión de la relación entre conceptos y en el razonamiento y juicio social frente a la solución de problemas cotidianos. 
Los procesos que en este momento afectan más la funcionalidad de la paciente, específicamente en lo que a su independencia respecta, son los que atañen al funcionamiento ejecutivo, donde, según la exploración neuropsicológica, la paciente presentó mayor gravedad en lo relacionado con la planificación, organización y ejecución de tareas necesarias para llevar acabo con éxito una actividad. Esto coincide una vez más con lo reportado en los estudios de Sorokin et al. [31] que sugieren que los tubérculos localizados en los lóbulos frontales pueden generar disfunción en los procesos de planeación, organización, síntesis y control. Por otra parte, las manifestaciones clínicas de esta enfermedad están relacionadas con enfermedades psiquiátricas, específicamente con la ansiedad y la depresión. De hecho, investigaciones como la de Tsai y Sahin [33] sugieren que los déficits en las conexiones cerebelosas pueden contribuir a los síntomas neuropsiquiátricos encontrados en el CET. Sin embargo, en este caso específicamente, la paciente B no presenta síntomas significativos que hagan pensar en una posible comorbilidad.

Finalmente, se encuentra en la exploración neuropsicológica que, si bien el funcionamiento cognitivo de B presenta limitaciones significativas, la paciente puede realizar tareas y operaciones básicas en la mayoría de los dominios cognitivos, lo cual le brinda una oportunidad de ir mejorando en su conducta adaptativa, que entendemos como un sistema multidimensional que incluye habilidades conceptuales, sociales y prácticas (actividades de la vida diaria, cuidado personal, manejo del dinero, entre otras). Es por esta razón que se plantea una intervención neuropsicológica enfocada en el funcionamiento ejecutivo dorsolateral.

\section{Conclusiones}

La Discapacidad Intelectual en el CET parece tener una incidencia alta (este estudio coincide con dicha presunción); sin embargo, la literatura reporta que no todos los pacientes con diagnóstico de GET y epilepsia tienen DI, por lo cual se hace necesario la exploración del funcionamiento cognitivo a través de la evaluación neuropsicológica, caracterizando de manera precisa los puntos fuertes y las dificultades que presenta la persona. Al comparar el desempeño cognitivo de la paciente con lo reportado por la evidencia científica, se observó que existen unos puntos de encuentro en lo que concierne a dominios con mayor afectación. En este caso, B presentó mayor dificultad en sus habilidades perceptuales, la velocidad de procesamiento, la memoria de trabajo, el lenguaje y el funcionamiento ejecutivo, lo que coincide, una vez más, con lo registrado. Cabe resaltar que, por ser un estudio de caso, los resultados de este no pueden ser generalizados. Lo que aportaría a otras exploraciones con una muestra de sujetos con características similares y con diagnóstico de CET es la propuesta de intervención neuropsicológica que pudiese ser probada en un contexto de investigación, ya que está fundamentada en tres modelos teóricos sólidos. Es importante avanzar en estudios en este tipo de patología con el fin de determinar, clarificar y conocer -en una muestra mas amplia de sujetos con diagnóstico similar al perfil cognitivo encontrado en el nuestro- la eficacia del protocolo de rehabilitación expuesta en este informe, lo que contribuiría al cuerpo teórico para la comprensión y abordaje del CET. 


\section{Referencias}

1. Boronat S, Sábado C, Vendrell T. Complejo esclerosis tuberosa, grupo de trabajo sobre cáncer en síndromes genéticos polimalformados. Madrid: Síntesis, 2015; p. 8-9.

2. Winterkorn, Pulsifer, Thiele. Cognitive prognosis of patients with tuberous sclerosis complex. Neurology [Internet]. 2007 Ene 2;68(1):62-68. DOI: https://doi.org/10.1212/01. wnl.0000250330.44291.54

3. Morales R. Perfil clínico de manifestaciones neurológicas de pacientes con Esclerosis Tuberosa y astrocitoma subependimario de células gigantes (Tesis para optar por el título de especialista en neurología) México: Universidad Nacional Autónoma de México, Facultad de Medicina; 2014.

4. Arango J, Delgado J, Saldarriaga W. Esclerosis tuberosa, diagnóstico fetal y materno. Rev chil obstet ginecol [Internet]. 2015 Dic;80(6):475-480 DOI: https://doi.org/10.4067/ S0717-75262015000600007

5. Arnedo, Montes, Bembible, Triviño. Neuropsicología infantil. Madrid: Panamericana; 2015.

6. Rosselli M, Matute E, Ardila A. Neuropsicología del desarrollo infantil. México: Manual Moderno; 2010.

7. Maulik PK, Mascarenhas MN, Mathers CD, Dua T, Saxena S. Prevalence of intellectual disability: a meta-analysis of population-based studies. Research in Developmental Disabilities. 2011 Mar-Abr; 32:419-436 DOI: https://doi.org/10.1016/j.ridd.2010.12.018

8. Fernández C. Estudio longitudinal del perfil neuropsicológico de adultos con discapacidad intelectual con y sin síndrome de down (Tesis doctoral). Madrid: Universidad Autónoma, Facultad de psicología, Departamento de psicología biológica y de la salud; 2013. Disponible en: https://repositorio.uam.es/handle/10486/14312

9. Sondenaa E, Rasmussen K, Nottestad, J, Lauvrud C. Prevalence of intellectual disabilities in Norway: domestic variance. Journal of Intellectual Disability Research [Internet]. 2010 Ene 18;54(2):161-167. DOI: https://doi.org/10.1111/j.1365-2788.2009.01230.x

10. Esteba CS, Peña CJ, García AJ, Castellanos, Torrents RD, Rodríguez E, Al E. Test Barcelona para discapacidad intelectual: un nuevo instrumento para la valoración neuropsicológica clínica de adultos con discapacidad intelectual. Rev Neurol. 2017 May 16;64(14):433444. DOI: https://doi.org/10.33588/rn.6410.2016400

11. Vries V, Veltman J. Genomic microarrays in mental retardation: from copy number variation to gene, from research to diagnosis. Med Genet [Internet]. 2010;47(5):289-297 DOI: https://doi.org/10.1136/jmg:2009.072942

12. Sharma P, Rao K. Psychological intervention in tuberous sclerosis: a case report. Indian Journal of Psychiatry. 2002;66(8):391-396 Disponible en: http://wwwindianjpsychiatry.org $/$ article. asp? issn $=0019-5545$; year $=2002$; volume $=44$; issue $=4$; spage $=391 ; \mathrm{e}-$ page $=396$; aulast $=$ Sharma; type $=0$

13. García J, Esteba S, Viñas M. Neuropsicología de la discapacidad intelectual de origen genético. Madrid: Sintesis; 2018. 
14. Echavarría LM. Modelos explicativos de las funciones ejecutivas. Revista de Investigación en Psicología [Internet]. 2017 Ago 18;20(1): 237-247. DOI: https://doi.org/10.15381/ rinvp.v20i1.13534

15. Danielsson H, Henry L, Messer D. Rönnberg J. Strengths and weaknesses in executive functioning in children with intellectual disability. Research in Developmental Disabilities [Internet]. 2012 Mar-Abr;2(33):600-607. DOI: https://doi.org/10.1016/j. ridd.2011.11.004

16. Muñoz M, Tirapu J. Rehabilitación neuropsicológica. Madrid: Editorial Síntesis S.A.; 2001.

17. Cárdenas DG, Rojas AF, Guellar S, Guella N. Estrategias de estimulación cognitiva para la mejora de la atención en adultos con diagnóstico de discapacidad intelectual. Psicoespacios [Internet]. 2017 Jul-Dic;1 1(19):3-23. DOI: https://doi.org/10.25057/21452776.949

18. Torres D, Galvis A, Lopera A, Montoya D. Función ejecutiva y entrenamiento computarizado en niños de 7 a 12 años con discapacidad intelectual. Rev. Chil. Neuropsicol. 2017 Dic 12;12(2):14-19.Disponible en: https://www.redalyc.org/pdf/1793/179354005002.pdf

19. Martínez M. Conocimiento científico general y conocimiento ordinario. Cinta moebio [Internet]. 2006;27:219-229 Disponible en: https://www.moebio.uchile.cl/27/martinez.html

20. Muñoz J, Quintero J, Munévar R. Cómo desarrollar compentencias investigativas en educación. Bogotá: Magisterio editorial; 2005.

21. Montero D. Evaluación de la conducta adaptativa en personas con discapacidades. adaptación y validación del icap. Bilbao: Mensajero; 1996.

22. Rey A. Rey: Test de copia y de reproducción de memoria de figuras geométricas complejas. Madrid: TEA ediciones; 1997.

23. Wechsler D. Manual for the wechsler adult intelligence scale-third edition. San Antonio, TX: Psychological Corporation; 1987.

24. Amador J. La Escala de memoria de Wechsler. 4 ed.(WMS-IV). Barcelona: Facultad de Psicología. Universidad de Barcelona; 2015

25. Renzi E, Faglioni P. Normative data and screening power of a shortened version of the Token Test. Revista Cortex [Internet]. 1978 Mar;14(1):41-49 DOI: https://doi. org/10.1016/S0010-9452(78)80006-9

26. Kaplan EF, Goodglass H, Weintraub S. the Boston naming test. 2 ed. Philadelphia: Lea \& Febiger; 1983

27. Luckasson R, Borthwick-Duffy S, Buntix WHE, Coulter DL, Graig EM, Reeve A, Cols. Mental Retardation: definition, classification and systems of supports. 10 ed.Washington, DC: American Association on Mental Retardation; 2002

28. Flores JC, Ostrosky F. Desarrollo neuropsicológico de lóbulos frontales y funciones ejecutivas. México: Manual Moderno; 2012.

29. Sholberg MM, Mateer CA, Stuss DT. Contemporary approaches to the management of executive control dysfunction. Journal of Head Trauma Rehabilitation [Internet]. 1993 Mar;8:45- 8. DOI: https://doi.org/10.1097/00001199-199303000-00006 
30. Ruggieri V, Arberas C. Fenotipos conductuales. Patrones neuropsicológicos biológicamente determinados. Rev neurol [Internet]. 2003;37:239-253 DOI: https://doi. org/ 10.33588/rn.3703.2003134

31. Sorokin I, Remes Lenicov MC, Pereira RV. Complejo esclerosis tuberosa: diagnóstico en la edad adulta. Arch Argent Dermatol [Internet]. 2015;65(3):104-109. Disponible en: http://www.archivosdermato.org.ar/wp-content/uploads/2019/10/104Sorokin-Complejo-esclerosis-tuberosa.pdf

32. Gómez MT, Vargas JA. Esclerosis tuberosa. Revista Médica Sinergia [Internet]. 2019 Mar;4(3):21-37 DOI: https://doi.org/10.31434/rms.v4i3.182

33. Tsai P, Sahin M. Mechanisms of neurocognitive dysfunction and therapeutic considerations in tuberous sclerosis complex. Curr Opin Neurol [nternet]. 2011 Abr;24(2):106113. DOI: https://doi.org/10.1097/WCO.0b013e32834451c4 